\title{
All research needs to follow the rules set down by Good Clinical Practice
}

\author{
Inge-Marie Velstra ${ }^{1} \cdot$ Angela Frotzler $^{1}$
}

Received: 17 June 2020 / Accepted: 19 June 2020 / Published online: 2 July 2020

(c) International Spinal Cord Society 2020

All researchers are obliged to follow the very detailed principles/rules of the International Conference on Harmonization-Good Clinical Practice (GCP). GCP is an international ethical and scientific quality standard for designing, conducting, recording, and reporting studies that involve the participation of human participants [1]. GCP is particularly important for clinical trials.

In investigator-initiated trials, the investigator (or institution) has to assume the sponsor responsibility. Main aspects of sponsor responsibilities are implementing a quality management system on an organizational and study level, risk management, data management, teaching/training, and risk-based monitoring [1]. Thus, the sponsor has to address many different GCP requirements. However, since GCP tells us just "what to do" but not "how to do it", the implementation of GCP often leads to frustration mainly due to its complexity, strictness and comprehensiveness. In our experience, study teams often consider the GCP guidelines as very burdensome to implement. However, fulfilling the GCP requirements is only our duty while doing clinical research but also a valuable guide to ensure participants' rights, well-being and safety, as well as data quality.

In the past, traditional monitoring was mostly based on frequent on-site visits and source data verification (e.g. checking Case Report Form data against source documentation, i.e. in patient charts). There is evidence that $100 \%$ source data verification during monitoring is less useful than originally thought, recognizing that not all clinical studies require the same monitoring approach and extent to ensure data quality or patient safety. Therefore in 2016, the concept of risk-based monitoring was introduced in the addendum to the International Conference on

Inge-Marie Velstra

inge-marie.velstra@paraplegie.ch

1 Clinical Trial Unit, Swiss Paraplegic Centre, Nottwil, Switzerland
Harmonization-GCP E6 (R2) guideline. This requires the sponsor to develop a systematic, prioritized, risk-based approach to monitoring clinical trials [1]. In risk-based monitoring, the risk of GCP deviations is assessed for a certain study center and type of clinical study. If the risk is high, frequent on-site monitoring visits are required, and if the risk is low, minimal on-site visits are scheduled. Hence, in risk-based monitoring the extent and nature of monitoring is flexible, and the goal is to improve the effectiveness and efficiency of monitoring. However, it is not always clear how to set up risk-based monitoring for clinical research projects. The establishment of an appropriate monitoring plan is still a challenge, although monitoring is simplified with the concept of risk-based monitoring. Thus, we recommend involving an experienced person in risk-based monitoring, for support with the writing of a monitoring plan according to the institutional conditions (like financial resources or existence of a quality management system).

There are various risk-based monitoring tools available $[2,3]$. In our institution, risk-based monitoring is generally based on the concept of Risk ADApted MONitoring [4] and is applied in both interventional and observational studies. Our common findings (i.e., GCP deviations) during monitoring visits over the last years were:

(1) Issues with informed consent: e.g., investigator signed the informed consent prior to the study participant; (2) protocol deviations: e.g., participants who do not meet the eligibility criteria are included; (3) issues with essential documents: e.g., no delegation log and thus no allocation of study tasks, missing or erroneous standard operating procedures; and (4) issues with Case Report Forms/source data: e.g., missing or erroneous, not readable data entries and inconsistencies.

Our risk-based monitoring experience indicated many GCP deviations [5, 6] that reflected the operationalization know-how of the study team. In general, the occurrence of minor findings during on-site monitoring visits in clinical research was high. However, there were no findings 
regarding safety, and no issues regarding electronic data capture. The collected data were mostly identifiable, recorded contemporaneously, and accurate. Overall, findings did not differ markedly between interventional and observational studies. Unfortunately, findings regarding participant rights, documentation, and essential documents continued to occur.

In our experience, risk-based monitoring indeed reduces monitoring time and efforts. However, in less experienced study teams, reduced monitoring can result in reduced data quality or late detection of protocol deviations. Extensive changes to the study processes or amendments of study protocol or documents after the inclusion of participants will nullify the time and efforts gained by risk-based monitoring. Therefore, even if the risk of a study is low, we recommend planning a monitoring (initiation) visit prior to the start of the study as well as after the inclusion of the first 1-3 participants. This will allow to assess and adapt study processes early on, and thus will increase the quality of the research performance of a study team, in the sense of a "learning research system".

Indeed, early monitoring visits (including initiation visits) can alert the study team of relevant obstacles, shortcomings or forecast possible problems, give prompt feedback and guidance in GCP. If necessary, it is also possible to train the study team in some aspects of GCP and study related tasks. Furthermore, the monitor can work with the study teams to prepare preventive steps to keep potential risks during the course of the study low. This will improve the conduct of a study, save time, reduce workload and costs and as a result, decrease the number of findings (i.e., GCP deviations) later on.

It is difficult to anticipate what might happen in the future over the course of a study. Independent monitoring is crucial in order to provide operational feedback regarding the study protocol and all other related documents.
We, therefore, recommend using monitoring in general as a preventive tool in clinical research. The application of monitoring should already be considered and specified during the planning phase of a study, to maximize the benefits from monitoring activities.

In all, there are many aspects and complexities to GCP. Researchers need to give these issues careful consideration, in order to ensure research integrity and that the safety and rights of all participants are respected.

\section{Compliance with ethical standards}

Conflict of interest The authors declare no conflict of interest.

Publisher's note Springer Nature remains neutral with regard to jurisdictional claims in published maps and institutional affiliations.

\section{References}

1. Guideline for Good Clinical Practice E6 (R2). Geneva: ICH (International Conference on Harmonisation); 2016. https://www. ich.org/fileadmin/Public_Web_Site/ICH_Products/Guidelines/ Efficacy/E6/E6_R2_Step_4.pdf.

2. ECRIN, European Clinical Research Infrastructure Network. Riskbased monitoring toolbox. Paris: ECRIN; 2016. https://www.ecrin. org/tools/risk-based-monitoring-toolbox.

3. Hurley C, Shiely F, Power J, Clarke M, Eustace JA, Flanagan E, et al. Risk based monitoring (RBM) tools for clinical trials: a systematic review. Contemp Clin Trials. 2016;51:15-27.

4. Brosteanu O, Houben P, Ihrig K, Ohmann C, Paulus U, Pfistner B, et al. Risk analysis and risk adapted on-site monitoring in noncommercial clinical trials. Clin Trials. 2009;6:585-96.

5. Beever D, Swaby L. An evaluation of risk-based monitoring in pragmatic trials in UK Clinical Trials Units. Trials. 2019;20:556.

6. von Niederhausern B, Orleth A, Schadelin S, Rawi N, Velkopolszky M, Becherer C, et al. Generating evidence on a risk-based monitoring approach in the academic setting - lessons learned. BMC Med Res Methodol. 2017;17:26. 